\title{
PHOTOIONIZATION EFFECTS ON GALAXY FORMATION
}

\author{
M. CHIBA \\ Astronomical Institute, Tohoku University, Sendai 980-77, Japan \\ AND
}

B.B. NATH

IUCAA, Post Bag 4, Ganeshkhind, Pune 411007, India

\begin{abstract}
We investigate the effects of the observed UV background radiation on galaxy formation. The biasing mechanism by photoionization is examined in detail, and its implication for galaxy number counts is discussed. Photoionization by UV radiation, $J$, decreases the cooling rate of the gas in halos, so that objects with only large density contrasts can selfshield against the radiation. In the context of the CDM model, we use the criterion that self-shielding is essential for star formation to calculate the mass function of galaxies (see Chiba \& Nath 1994, ApJ, 436, 618 for details). We find that the cooling rate in the big (low-density) system, into which smaller objects are merged, is reduced by photoionization. This means that, in a merging dominated region, where the number density at the low-mass end $\left(M_{b} \leq 10^{10} M_{\odot}\right)$ is usually expected to decrease with time, the trend is reversed (the number of low-mass galaxies is more at lower redshifts $z$ ) due to the decreasing UV flux with time after $z \sim 2$ (see Figure 1).
\end{abstract}
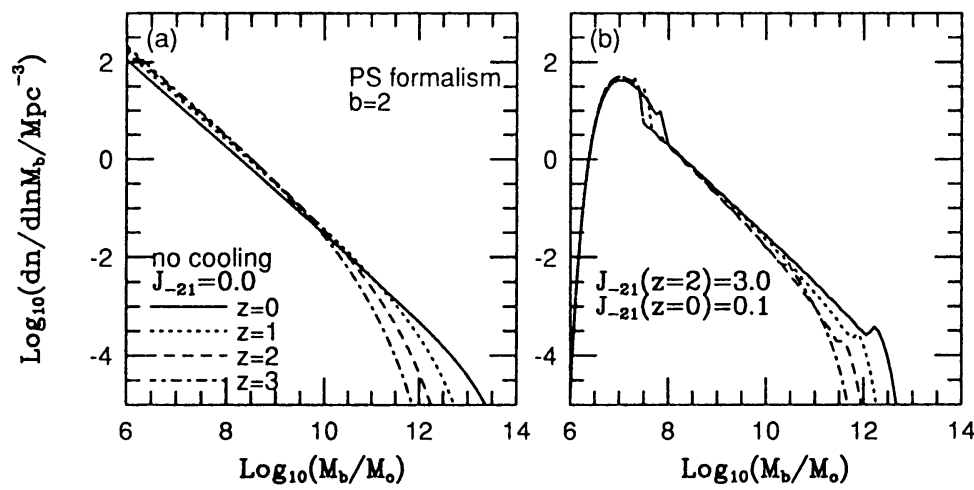

Figure 1. The Press-Schechter mass functions. (a) $J=0$ (b) $d J / d z>0$ 